\title{
Anticholinergic-induced analgesia: possible role for the cholinergic system in abnormal sensory symptoms in Parkinson's disease
}

\author{
Reuven Sandyk
}

Department of Internal Medicine (Neurology), The University of Arizona, Tucson, Arizona, USA.

\begin{abstract}
Summary: Sensory symptoms related to pain perception have been reported to occur in $30-50 \%$ of parkinsonian patients. Two patients with Parkinson's disease are reported, in whom painful sensory phenomena preceded or accompanied the disease process. In both patients the sensory phenomena were unresponsive to therapy with oral narcotics, anti-inflammatory drugs or administration of levodopa/ carbidopa. Benzhexol (4-6 mg/day) produced dramatic amelioration of symptoms, indicating a role for the cholinergic system in the pathophysiology of abnormal sensory symptoms in Parkinson's disease and possibly in human analgesia in general.
\end{abstract}

\section{Introduction}

Sensory symptoms related to pain perception have been reported to occur in $30-50 \%$ of parkinsonian patients (Coyle \& Snyder, 1969). In the majority of cases, patients experienced recurrent pain and other abnormal sensations that could not be attributed directly to disorders of the musculoskeletal or vascular systems. Furthermore, these abnormal sensations did not appear to be related to the presence of the motor disabilities such as rigidity or tremor (Snider et al., 1976). It is thus believed that the abnormal sensory phenomena experienced by these patients were caused primarily by the disease process itself, and were not secondary to any of the motor malfunctions of the disease.

The pathogenesis of the abnormal sensory symptoms experienced by some parkinsonian patients is not well understood. Nutt \& Carter (1984) have recently suggested a role for the dopaminergic system in the pathogenesis of these symptoms. Administration of Ldopa during the 'off' period produced amelioration of distressing sensory phenomena in 2 patients with Parkinson's disease, while Snider et al., (1976) and Sandyk (1982) reported amelioration of sensory phenomena with oral narcotic analgesics. The latter reports suggested a role for the endogenous opioid system in the pathogenesis of primary sensory symptoms in parkinsonism (Sandyk \& Snider, 1985). The report by

Correspondence: R. Sandyk, M.D., M.Sc. Department of Internal Medicine Arizona Health Sciences Center Tucson, AZ 85724, USA.

Accepted: 24 January 1986
Snider et al. (1976) suggested, however, that not all cases of parkinsonism with abnormal sensory disturbances respond to either dopaminergic drugs or opiates, indicating a role for other transmitter systems in the pathogenesis of abnormal sensory phenomena in Parkinson's disease. The following report concerns 2 patients who experienced abnormal sensory phenomena related primarily to pain, in whom administration of anticholinergics (benzhexol) produced dramatic relief of symptomatology.

\section{Case reports}

Case 1

A 54 year old man presented with generalized pain of 12 months duration. The pain was described as dullaching and at times burning and involved predominantly the trunk, the lower back and muscles and joints of both upper extremities. These sensations were present throughout the day and night, were unrelated to movement, and were not relieved by standard analgesics, non-steroidal anti-inflammatory drugs or physical therapy. General examination was unremarkable. Neurological assessment revealed no evidence for musculoskeletal disease. However, he had some mild stigmata of parkinsonism that included facial amimia, positive glabellar tap, decreased arm swing, and mild hypokinesia and right-sided upper limb rigidity. The rest of the examination was normal. Laboratory investigations, including serum bio-

(C) The Fellowship of Postgraduate Medicine, 1986 
chemistry, creatine kinase, sedimentation rate, antinuclear factors, thyroid studies, vitamin $\mathbf{B}_{12}$ and folate, were normal or negative. Radiographs of the spine and shoulder regions were normal. Computed tomographic (CT) scan of the head was normal.

A 2 week trial with oral analgesics alone or combined with anti-inflammatory agents and physical therapy produced no relief. Administration of levodopa/carbidopa (Sinemet $10 / 100 \mathrm{mg}$ twice daily) produced improvement in the patient's mobility and he reported being less 'stiff' but his sensory phenomena were unchanged. Increasing the Sinemet dosage to 5 tablets/day produced no change in the patient's sensory complaints. He was then given a trial with benzhexol ( $2 \mathrm{mg}$ twice daily) that produced dramatic alleviation of his painful sensations within several days of initiation of therapy. Increasing the benzhexol dosage to a total of $6 \mathrm{mg} /$ day produced further improvement and he remained entirely free of pain for over a year. Attempts to reduce the dosage, discontinue the drug or replace it with placebo (pyridoxine $100 \mathrm{mg} /$ day) produced rapid recurrence of the painful sensations.

\section{Case 2}

Three years before presentation, this 62 year old woman experienced unpleasant sensations involving the lower abdomen, upper extremities and left lower limb. These sensations occurred intermittently, were unaffected by movement and were characterized by burning, tingling and aching sensations. They were present particularly during the day, but occasionally during the night. She also reported that her legs felt 'stiff' at times, but this had not affected her walking. There were no abnormal signs on examination and neurological investigations, including radiographs of the spine, chest, skull and abdomen, CT scan of the head, electroencephalogram and cerebrospinal fluid analysis were normal or negative. Serum biochemical investigations, including full blood count, glucose, liver and renal functions, creatine kinase and antinuclear factors, vitamin $B_{12}$ and sedimentation rate, were normal or negative. Administration of non-steroidal anti-inflammatory agents, prednisone and several oral narcotic agents produced no benefit.

Approximately one year later, she noticed gradual slowing of her walking and experienced occasional falls. On recent examination she had a mild resting tremor in her left upper limb, mild expressionless face, and reduced arm swing on the left during walking. There was some rigidity in the neck and wrist muscles, and the glabellar tap was positive. Tendon jerks were normal and plantar responses were flexor bilaterally. There was no sensory deficit and cerebellar signs were absent. She was put onto levodopa/carbidopa (Sinemet, $25 / 100 \mathrm{mg}$ twice daily), and her mobility and tremor improved considerably. The senory phenomena remained unchanged, however. She was then given, in addition to the Sinemet, benzhexol (Artane ${ }^{R}$, $2 \mathrm{mg}$ twice daily) that produced relief of her painful sensations within 48 hours. As she experienced no distressing anticholinergic effects, the dosage of benzhexol was raised to $2 \mathrm{mg}$ three times daily. On this dosage she experienced further improvement in her symptoms and remained almost free of pain over a 6 month period. Two trials to reduce the dosage of benzhexol to $1 \mathrm{mg}$ three times daily, or replace it with amantadine $(100 \mathrm{mg}$ three times daily) produced recurrence of her painful sensations within 72 hours.

\section{Discussion}

In these patients, as in other reported literature (Koller, 1984; Schott, 1985) painful sensory phenomena antedated or were present at the initial stages of the disease. These sensory disturbances improved occasionally with dopamine agonists but many patients experienced no relief with a variety of agents (Snider et al., 1976).

The mechanism(s) by which dopaminergic agents produced alleviation of painful sensory symptoms in parkinsonism are not understood. Dopamine has been shown to play a role in pain suppression (Akil \& Liebeskind., 1975) and to be efficacious in the therapy of pain in herpes zoster (Kernbaum \& Hauchecorneşa 1981) and cancer (Minton, 1974). Our patients failed, however, to respond to levodopa although other features of their motor disability improved, suggesting that the painful sensations were unrelated to the motor disability. Furthermore, their symptoms were resistent to administration of oral analgesics and non-steroidal anti-inflammatory drugs, suggesting a role for nonopioid mediated mechanisms in the pathophysiology of painful sensations in Parkinson's disease. The patient's dramatic response to benzhexol indicates a role for the cholinergic system in human analgesia. Indeed, the cholinergic system has been suggested to be involved in analgesia induced by morphine (Domino, 1975) and has also been implicated in modulating stress-induced analgesia in animals (Lewis \& Liebeskind, 1983). Furthermore, involvement of cholinergic mechanisms in certain human pain states has also received some support from a recent report demonstrating that anticholinesterase drugs are useful in the management of chronic pain (Schott \& Loh, 1984). Paradoxically, however, our patients experienced pain relief with anti-cholinergic medication.

Anti-cholinergic agents have been shown to be efficacious in the management of motor symptoms in Parkinson's disease. Apart from reducing the functional overactivity of cholinergic interneurones in the striatum, these agents have been shown to increase 
striatal dopamine levels by inhibiting dopamine reuptake (Coyle et al., 1969). However, since our patient's painful sensory phenomena failed to respond to Sinemet, it seems unlikely that the analgesic effects of benzhexol were mediated via dopaminergic mechan-

\section{References}

AKIL, H. \& LIEBESKIND, J.C. (1975). Monoaminergic mechanisms of stimulation produced analgesia. Brain Research, 4, 279.

COYLE, J.T. \& SNYDER, S.H. (1969). Antiparkinsonian drugs: inhibition of dopamine uptake in the corpus striatum as a possible mechanism of action. Science, 166, 899.

DOMINO, E.F. (1975). Role of central cholinergic mechanisms in the specific actions of narcotic agonists. In Cholinergic Mechanisms, Waser, P.G. (ed). pp. 433-453. Raven Press: New York.

KERNBAUM, S. \& HAUCHECORNE, J. (1981). Administration of levodopa for relief of herpes zoster pain. Journal of the American Medical Association, 246, 132.

KOLLER, W.C. (1984). Sensory symptoms in Parkinson's disease. Neurology, 34, 957.

LEWIS, J.W. \& LIEBESKIND, J.C. (1983). Pain suppression systems of the brain. Trends in Pharmacological Sciences, 4, 73 . isms. Whatever the mechanism(s) of benzhexol-induced analgesia may be, this report indicates a complex function of the cholinergic system in pain control and in the pathophysiology of abnormal sensory phenomena in Parkinson's disease.

MINTON, J. (1974). The response of breast cancer patients with bone pain to L-dopa. Cancer, 33, 358.

NUTT, J.G. \& CARTER, J.H. (1984). Sensory symptoms in parkinsonism related to central dopaminergic function. Lancet, ii, 456.

SANDYK, R. (1982). Back pain as an early symptom in Parkinson's disease. South African Medical Journal, 61, 3.

SANDYK, R. \& SNIDER, S.R. (1985). Sensory symptoms: Parkinson disease. Neurology, 35, 619.

SNIDER, S.R., FAHN, S., ISGREEN, W.P. \& COTE, L.J. (1976). Primary sensory symptoms in parkinsonism. Neurology, 26, 423.

SCHOTT, G.D. (1985). Pain in Parkinson's disease. Pain, 22, 407.

SCHOTT, G.D. \& LOH, L. (1984). Anticholinesterase drugs in the treatment of chronic pain. Pain, 20, 201. 